Revista de Investigación Educativa 27

julio-diciembre, 2018 | ISSN 1870-5308 | Xalapa, Veracruz

Instituto de Investigaciones en Educación | Universidad Veracruzana

\title{
Formación y uso de TIC en educación superior: opiniones del profesorado
}

\section{Training and use of ICT in higher education: teachers opinions}

\author{
José Ricardo López Espinosa ${ }^{a}$ \\ Edgar Oswaldo González Bello ${ }^{\text {b }}$ \\ Rocío López González \\ Recibido: 18 de agosto de 2017 \\ Aceptado: 10 de julio de 2018
}

En este trabajo se analizan las deficiencias y avances que se han generado en el marco del Programa Institucional de Formación Docente de la Universidad de Sonora y su contribución al desarrollo de la enseñanza mediante la capacitación del profesorado sobre el uso de Tecnologías de Información y Comunicación en la enseñanza. Es un estudio de corte cuantitativo en el cual se aplicó un cuestionario a 80 profesores, de distintas disciplinas, quienes han participado en cursos de capacitación que ofrece la institución para el uso de recursos digitales. Los resultados revelan la necesidad de ofrecer capacitaciones acordes a los requerimientos particulares de la planta docente, así como cursos especializados enfocados en instruir cómo emplear pedagógicamente las tecnologías en cada asignatura con el propósito de mejorar el ejercicio docente. También se advierte que la formación ofrecida en la institución poco ha logrado motivar a los profesores para utilizar las tecnologías.

Palabras clave: Educación superior; formación docente; TIC.

\footnotetext{
a Estudiante de doctorado en Innovación Educativa, Universidad de Sonora, México. 凶 josericardoloes@gmail.com

b Doctor en Ciencias Sociales. Profesor-Investigador, Universidad de Sonora, México. 凶 edgar.gonzalezb@gmail.com

'Doctora en Pedagogía. Investigadora del Programa en Investigación e Innovación en Educación Superior, Universidad Veracruzana, México.凶r rociolopez@uv.mx
} 


\title{
Formación y uso de TIC en educación
}

superior: opiniones del profesorado

José Ricardo López Espinosa, Edgar Oswaldo

González Bello, Rocío López González

This paper analyzes the deficiencies and progress that has been generated under the Institutional Program of Teacher Training of the University of Sonora and its contribution to the development of teaching through teacher training on the use of Information and Communication Technologies in teaching. It is a descriptive study of quantitative methodology in which questionnaires were applied to 80 teachers from different disciplines who have participated in training courses offered by the institution the use of Іст. The results reveal the need for trainings according to the requirements of teachers, as well as the lack of specialized courses focused on instructing how to use the technologies in each subject for the purpose of improving the teaching performance. It is also noted that the training provided in the institution has not managed to motivate teachers to use the technologies.

Keywords: Higher education; teacher training; Іст.

\section{Formación y uso de TIC en educación superior: opiniones del profesorado}

\author{
Training and use of ICT in higher education: teachers opinions
}

\section{Introducción}

$\mathrm{D}$ esde sus inicios, las Instituciones de Educación Superior (IES) han proporcionado formación profesional y educación para los individuos, generando una conexión directa con las necesidades de la sociedad. Sin embargo, las funciones básicas en las universidades se han modificado debido a las nuevas exigencias que enfrentan, añadiendo la intención de ser emprendedoras para tener relevancia en el mercado actual (Altbach, 2008).

Debido a la aceptación de las Tecnologías de Información y Comunicación (TIC) en la sociedad, existe una exigencia de los estudiantes hacia los profesores acerca de incluir los recursos digitales como medios para el desarrollo de los procesos de ense- 
ñanza. A partir de esto, existe el reto de generar la capacidad en los profesores para el diseño de estrategias didácticas que, además de estar adaptadas a las condiciones de la institución, permitan ofrecer a los estudiantes formación en torno a cómo atender y resolver situaciones mediante el apoyo de las Tic. Tal situación, ha puesto a las universidades en la necesidad de revisar sus ofertas educativas para contemplar en sus planes y estructura organizativa cómo se van a incluir y/o aprovechar las tecnologías para la enseñanza (Ramírez \& Casillas, 2014).

En este sentido, las IES ofrecen capacitación a los profesores sobre el uso de TIC en el ejercicio docente, buscando innovar en los procesos de enseñanza y con el fin de transformar la concepción sobre las tecnologías en cuanto a la utilidad de éstas en la docencia, y así aprovechar las inversiones en infraestructura tecnológica (Ducoing \& Fortoul, 2013). Este trabajo presenta un estudio realizado en la Universidad de Sonora (UNISON), donde se analizan las deficiencias y avances, a partir de las opiniones de los profesores, que se han generado en el marco del programa de formación y capacitación docente sobre el uso de las Tic.

\section{Formación docente y uso de las TIC en las IES de México}

A nivel global, se han diseñado diversas políticas, reformas e iniciativas que proporcionan lineamientos y sugerencias, advirtiendo la pertinencia de incluir las TIC en la enseñanza universitaria, además de capacitar a los profesores para estar en condiciones de innovar en la docencia. Según Careaga y Avendaño (2007), la inversión en infraestructura tecnológica debe permanecer, aunada a la implementación de programas formativos que estén enfocados en desarrollar las competencias tecnológicas, considerando una nueva definición del desempeño profesional por los profesores universitarios.

La Organización para la Cooperación y el Desarrollo Económico (OCDE, 2002) ha planteado una serie de argumentos que respaldan la inclusión de las TIC en la educación, infiriendo una variedad de beneficios relacionados con diversos aspectos (económicos, sociales y pedagógicos) que buscan apoyar el desarrollo de la formación en los estudiantes. Por su parte, la Organización de las Naciones Unidas para la Educación, la Ciencia y la Cultura (UNESCO, 2004) enfatiza la pertinencia de emplear las TIC en la enseñanza y sugiere ofrecer a los profesores una formación docente que plantee a la educación como eje central al momento de instruir sobre el uso de TIC. 
En torno a esto, Swig (2015) recopila información sobre la formación tecnológica proporcionada en los países de América Latina y el Caribe, afirmando que en los últimos 25 años en estos países se han iniciado programas para incorporar las TIC en sus sistemas educativos, generando políticas para estimular innovaciones en la enseñanza del profesorado por medio del empleo de las tecnologías; sin embargo, no en todos los casos se ha tenido el éxito deseado y varios de estos países carecen de tácticas específicamente relacionadas con las TIC y la formación docente. También afirma que tales iniciativas han sido poco suficientes, ya que capacitar a los docentes en el uso básico de las TIC es solamente el primer paso para mejorar la calidad de la enseñanza. Por otro lado, se asegura, existen muchos profesores en Latinoamérica y el Caribe que carecen de una computadora en casa, lo que limita en gran medida la familiaridad del docente con la tecnología. Afortunadamente, esta tendencia está empezando a cambiar.

De tal forma, la Universidad ha implementado capacitaciones con el fin de generar en los profesores cambios que posibiliten el empleo de las Tic en el ejercicio docente de una manera innovadora. Fullan (2002), argumenta que al implementar un cambio, éste pasa por diferentes fases antes de poder generar resultados efectivos; la primer fase implica a los docentes, quienes analizan los objetivos que tiene tal cambio y posteriormente inician adoptando y ejecutando los cambios más cercanos a su entendimiento, lo cual representa la segunda fase; en la tercer fase los docentes se comprometen con los cambios y se involucran en la implementación; en la quinta fase, los docentes se motivan para continuar reproduciendo el cambio y se capacitan para ejecutarlo de la manera más efectiva posible, y en la sexta y última fase, los docentes conciben un cambio significativo involucrándose en el mismo y reproduciéndolo de la manera más eficiente logrando los resultados esperados.

Para el caso de la formación docente, Del Moral y Villalustre (2012) afirman que ésta es el elemento clave para facilitar la interacción de las TiC en la enseñanza, y que a su vez, debe favorecer el desarrollo y la adquisición de diferentes habilidades que permitan al docente utilizar didácticamente las herramientas y aplicaciones tecnológicas dentro del aula. Sin embargo, a pesar de que las tecnologías apropiadamente utilizadas tienen el potencial de modificar la enseñanza y mejorar el aprendizaje, a nivel global, tanto la creación de políticas para la integración de las TIC en los sistemas educativos como la inversión en infraestructura tecnológica en las universidades, no parecen haber producido los efectos esperados (Ducoing, 2003). 
Por otra parte, Correa y Martínez (2010) han identificado limitantes para una integración exitosa de las TIC en las universidades, agrupadas en tres diferentes niveles: micro (profesores), meso (instituciones) y macro (sistema). En el nivel referente al profesorado, las limitantes principales indican una falta de habilidades para el uso de TIC y la necesidad de una actualización constante para aprovechar las tecnologías en la enseñanza. Al nivel de las instituciones, además del acceso a las TIC y la infraestructura, es relevante incorporar a aquéllas dentro de la visión de las universidades, mediante una formación docente que favorezca el desarrollo de las competencias tecnológicas. En cuanto al sistema educativo, implica la necesidad de modificar y actualizar los métodos de evaluación en los profesores e instituciones, los cuales deben contemplar las habilidades adquiridas y requeridas al utilizar las TIC para el ejercicio docente.

Al indagar sobre la formación docente para el empleo de las TIC en la enseñanza universitaria, es evidente la relevancia de tomar en cuenta el contexto en el que se suscribe cada institución, pues presentan de manera diferente tanto las dificultades como los obstáculos. Para llevar a cabo el análisis aquí presentado, se observó al objeto de estudio desde tres aristas; una referente a la problemática que para el profesorado ha representado utilizar las TIC en la enseñanza; otra sobre la formación institucional docente en relación con el uso de TIC, y una visión general sobre la contribución de la política nacional en la enseñanza universitaria mediante el uso de tecnologías. Esto alude a los tres niveles sugeridos por Correa y Martínez (2010).

Desde una perspectiva internacional, existen organismos (UNESCO, OCDE, entre los principales) encargados de estudiar y diagnosticar diversas problemáticas que son percibidas en las IEs, sugiriendo, a su vez, aprovechar los beneficios que se generan con el desarrollo tecnológico para mejorar los procesos de enseñanza. Derivado de estos diagnósticos, en México se han diseñado las políticas que han planteado estrategias y lineamientos para alcanzar una educación de calidad, generando la posibilidad de innovar en el ejercicio docente mediante la inversión en infraestructura tecnológica y la formación docente para el aprovechamiento de tal inversión (Gobierno de la República, 2013).

Siendo así, las IES en México se encuentran influenciadas por diversas recomendaciones, pero principalmente por los lineamientos de la política nacional, donde el empleo de TIC para la enseñanza se presenta como parte del Plan Nacional de Desarrollo 2013-2018 (Gobierno de la República, 2013), a través de una política que presenta estrategias, metas y líneas de acción para la concepción de una educación de 
calidad, la cual es planteada como un avance que permitirá la posibilidad de innovar en la enseñanza. Dicha política hace hincapié en la pertinencia de favorecer a las universidades mediante la inversión en infraestructura, proponiendo incluir a las TIC para innovar en la enseñanza. También se presenta una línea de acción en torno a la formación de docentes para el desarrollo de las habilidades tecnológicas, otorgando la posibilidad a las instituciones de elegir el tipo de formación que se ofrecerá a los profesores, y permitiendo la diversidad en cuanto a contenidos y cantidades de cursos que pone a disposición cada institución. De esta manera, cada IEs desarrolla sus funciones ante diversas recomendaciones, ofreciendo formación al profesorado para el uso de la infraestructura tecnológica y retomando tanto los lineamientos de la política como las sugerencias que otorgan los organismos para articularlas con su misión y visión institucional.

Por otro lado, también puede ser apoyado el mejoramiento de la enseñanza por la participación de algunos profesores en programas de estímulos que evalúan el desempeño docente, por lo que pueden contar con diferentes características que contribuyan con el desarrollo de habilidades para el empleo de las Tic. Por ejemplo, un profesor que ha adquirido financiamiento para equipamiento tecnológico puede requerir habilidades diferentes que a quien no se le ha apoyado mediante este tipo de incentivo. Por tanto, y debido a que varios de estos programas se derivan de las sugerencias de las políticas nacionales, se infiere la pertinencia de tomar en cuenta la relación de los objetivos de estas políticas con las habilidades requeridas en los profesores, de acuerdo con la participación en el contexto institucional mediante el empleo de las Tic.

Pero ¿en qué medida se podría afirmar que la formación docente en las universidades ha logrado contribuir al desarrollo de la educación de calidad que se ha propuesto? López (2013) argumenta que cada universidad sigue un camino propio, acorde a su visión y misión institucional, además del modelo educativo, por lo que se debe reconocer a cada institución como un establecimiento representativo de una variedad de casos complejos y particulares. Al analizar la formación docente, se requiere tomar en cuenta los objetivos de cada universidad, su contexto y su relación con las intenciones de las políticas para establecer qué tan distante se encuentran de lograr los objetivos planteados. En tanto, López y Ramírez (2016) consideran fundamental analizar el aprovechamiento que la comunidad académica le da a la infraestructura tecnológica de las IES, ya que generalmente el impulso que se le realiza a través de las políticas para el uso del equipamiento en las instituciones suele carecer de la exigencia de una 
previa reflexión. De ahí que cada universidad puede plantear diferentes estrategias de formación para el aprovechamiento de las TIC y, por lo tanto, obtener resultados que pueden diferir o no de los objetivos de las políticas.

En síntesis, una de las principales preocupaciones a nivel global en relación con el desarrollo de la enseñanza en las universidades, apunta a la necesidad de una mayor inversión en infraestructura tecnológica para impulsar mejoras en la calidad de la educación (UNESCO, 2006). Sin embargo, se retoma a Salado, Velázquez y Ochoa (2014), quienes sugieren que implementar las TIC en una institución educativa no es una tarea sencilla, pues para que tal implementación pueda tener mayores probabilidades de éxito, es necesario un gran esfuerzo de parte de los dirigentes de la institución, ya que ellos deben conocer las limitaciones y los beneficios de la enseñanza mediante el empleo de las tecnologías, y de esta manera llevar a cabo estrategias conscientes sobre lo que se puede y debe lograr.

Si bien se ha sugerido la inversión en infraestructura tecnológica como un medio para ampliar las posibilidades de mejorar la enseñanza mediante el uso de las TIC (UNESCO, 2004), esta inversión se debe pensar en función de las condiciones institucionales, en tanto que lo invertido se pueda implementar en un contexto favorable para producir los resultados esperados. Así mismo, los profesores deben recibir una formación que les permita la posibilidad de mejorar su ejercicio docente mediante el uso de la infraestructura tecnológica en la que se llegue a invertir. Esto debido a las necesidades y exigencias de los estudiantes del siglo XXI, que orillan a los profesores a buscar el desarrollo de habilidades y saberes que permitan la apropiación y el uso de las tecnologías como estrategia para el empleo eficiente de las TIC para la enseñanza, ya que el solo hecho de adquirir equipamiento de infraestructura tecnológica, no garantiza que se sepa utilizar (Torres \& Barona, 2012).

De esta manera, se presenta el caso de la UNISON, una institución educativa del sector público que desempeña sus funciones buscando ser una IES innovadora: en el rubro tecnológico, se han favorecido las aulas y los espacios por medio de equipamiento tecnológico que constituye uno de los recursos primordiales para el mejoramiento de los servicios educativos (Grijalva, 2012).

Según su Plan de Desarrollo Institucional (PDI) 2013-2017 (UNISON, 2013), la institución mantiene como acción un programa de capacitación que aborda aspectos disciplinarios, didácticos y pedagógicos, el cual tiene como uno de sus propósitos proporcionar formación a los profesores para mejorar la calidad de su ejercicio docente, mediante la generación de habilidades para el uso de TIC. Cabe mencionar que en 
dicho programa de capacitación se espera una participación reflexiva por parte de los profesores y una actitud entusiasta en el ejercicio docente.

Por otro lado, también se indica que en 2013 la meta fue capacitar a 150 docentes, así mismo, se plantea que otra meta es duplicar la cantidad a 300 profesores capacitados para el año 2017. Esto, permite concebir una capacitación que busca instruir a un número de población limitada con relación a los 2,516 profesores adscritos a la institución; 1,274 son profesores de asignatura y 1,242 de carrera ${ }^{1}$ (UNISON, 2013). Cabe señalar que los profesores tienen la oportunidad de acudir voluntariamente a recibir formación del Programa Institucional de Formación Docente (PIFD), y no se hace de manera diferenciada, es decir, de acuerdo con las necesidades de cada disciplina.

En este sentido, y a pesar de que el PIFD vincula algunas intenciones con el PDI vigente, referidas a desarrollar las competencias TIC en los profesores por medio de la formación, se puede reconocer que sus metas no se relacionan del todo. Si bien en el PIFD se plantea formar profesores entusiastas que utilicen las tecnologías no sólo para comunicar información sino también que promueva el trabajo colaborativo entre los alumnos (UNISON, 2015), la institución espera contar con menos del 10\% de profesores capacitados, lo cual es poco (si se compara con el total de la planta académica). Aunado a ello, la información sobre la planta académica en torno al acceso y uso de la infraestructura tecnológica parece ser insuficiente para establecer un diagnóstico concluyente; sin embargo, se ha sugerido llevar a cabo estudios relacionados con el interés de los profesores por capacitarse, así como de revisión sobre la pertinencia del programa de formación institucional para el ejercicio docente (Borbón, 2015; Leyva, 2015).

\section{Método}

Para el desarrollo de este trabajo se contempló analizar el objeto de estudio desde un paradigma positivista, el cual sustenta que existe una realidad social fuera del individuo que es objetivamente conocible (Corbetta, 2007); además, permite observar el contenido temático de una disciplina o de un fenómeno determinado a través de la asociación con determinadas metodologías (Sautu, Boniolo, Dalle \& Elbert, 2005).

1. 1,091 son profesores e investigadores de tiempo completo, nueve de medio tiempo, 140 técnicos académicos de tiempo completo y dos de medio tiempo (UNISON, 2013). 
Debido al interés por conocer las opiniones de los profesores con relación al tipo de formación que reciben (para el empleo de las TIC), y las condiciones de infraestructura tecnológica con la que cuentan, se consideró un método cuantitativo, ya que éste permite comprender la realidad objetiva del fenómeno sin profundizar necesariamente en cuestiones subjetivas (Tamayo, 2011). Particularmente, se trabajó con profesores de la UnISON adscritos a la Unidad Regional Centro que acreditaron al menos un curso de capacitación en el área informática y tecnológica, los cuales se ofrecieron como parte del PIFD entre 2013 y 2015.

Para la recolección de datos se aplicó un cuestionario, el cual se construyó a partir del instrumento Survey in Schools: ICT and Education, que fue desarrollado con base en las concepciones de Starkey, Law, Pelgrum y Plomp, Balanskat y Gertsch, y OCDE (European Union, 2013) en temas relacionados con las competencias digitales para la educación escolarizada. ${ }^{2}$

En cuanto a la validación del instrumento, se elaboraron dos tipos de análisis: $\mathrm{RASCH}$ y factorial exploratorio, los cuales permitieron determinar los reactivos que los sujetos son capaces de responder sin problemas, así como definir escalas en función de la relación entre los ítems de cada dimensión que midió el cuestionario. Además, se calculó el alfa de Cronbach, cuyos resultados indicaron un coeficiente que está cerca o es mayor a .75 en cada reactivo.

De esta forma, el instrumento se conformó por 24 reactivos que fueron organizados en dos dimensiones: a) Condiciones de infraestructura tecnológica institucional, orientada a la indagación de las opiniones de los docentes sobre las condiciones de infraestructura tecnológica de la institución y los obstáculos que para ellos representa su uso (Altbach, 2008; OCDE, 2002; Ramírez \& Casillas, 2014; UNESCO, 2006) y b) Formación institucional docente, referente a la indagación de las opiniones de los docentes en torno a la formación docente, los estímulos que reciben para participar en esta, la utilidad que consideran tiene la formación para el mejoramiento de habilidades que consideran necesitar en torno a cuestiones pedagógicas y tecnológicas (Ducoing \& Fourtoul, 2013; Marcelo, 2013; Tearle, 2003; UNESCO, 2004).

De los profesores que cumplieron con el criterio de haber acudido al menos a un curso de formación de los ofrecidos en el PIFD (186 profesores), se logró aplicar el

2. El cuestionario originalmente fue diseñado para aplicarse en niveles educativos inferiores al de la educación superior, por lo que se realizaron adaptaciones referentes al contexto mexicano y al escenario local de la institución. 
instrumento de manera impresa a un total de 80 ( $3 \%$ de la población total de profesores en la institución), quienes están adscritos a los diferentes departamentos de la Unidad Regional Centro de la UnISON. De estos, $44 \%$ fueron hombres y $56 \%$ mujeres. De ellos, el $16 \%$ se encontró entre un rango de edades menor a 45 años, $35 \%$ tiene entre 45 y 55 años y $49 \%$ ha alcanzado más de 55 años. De acuerdo con los datos, quienes mayormente participan en el PIFD son los profesores de más edad y con mayor tiempo de experiencia como docentes en la institución; sin embargo, no participan en el PIFD la gran mayoría de docentes adscritos a la institución (en total, 2,559).

Como se puede ver en la Figura 1, la proporción de sujetos que además de recibir capacitación sobre TIC también participa en programas de incentivos para el desarrollo de su ejercicio docente, como el Programa para el Desarrollo Profesional Docente (PRoDep) o el Programa de Estímulos al Desempeño del Personal Docente (PEDPD), es mayor a la proporción de profesores que participan en el PIFD y han sido reconocidos por el Sistema Nacional de Investigadores (SNI).

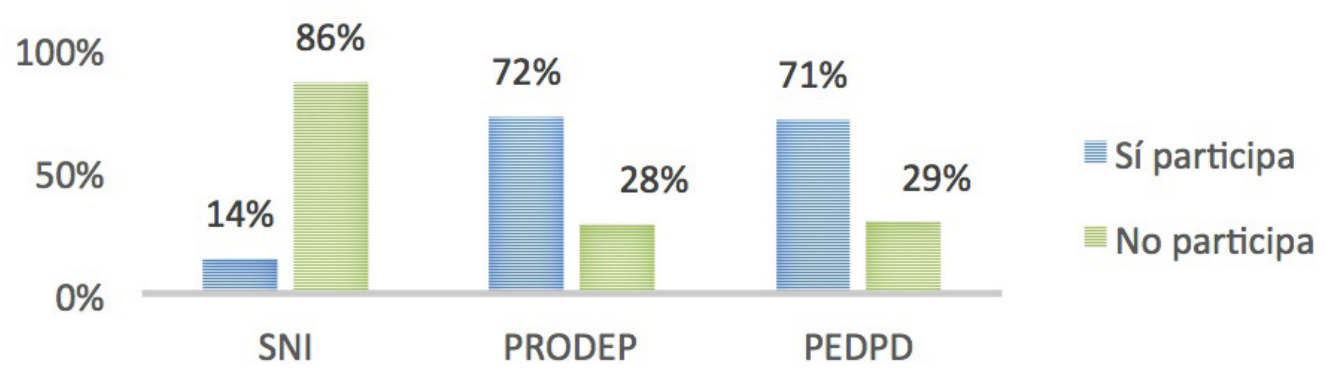

Figura 1. Participación de profesores encuestados en programas de incentivos

Fuente: Elaboración propia.

La cantidad de profesores presentada en la Tabla 1 es con relación a los datos obtenidos a partir de las encuestas aplicadas a los 80 sujetos que han participado en el área informática-tecnológica del PIFD recibiendo algún curso; sin embargo, la cantidad total de profesores apoyados por los programas de incentivos a nivel institucional es la siguiente: PRODEP, 566 profesores; PEDPD, 490 profesores, y SNI, 303 profesores. De tal forma que de los adscritos al PRODEP solamente $10 \%$ ha participado en el PIFD, de 
los adscritos al sin, 4\%, y de los adscritos al PEDPD, 12\% ha decidido recibir la capacitación de algún curso sobre TIC en el PIFD.

Estos datos se relacionan con la utilidad que tiene la formación institucional sobre TIC para los profesores, ya que los apoyos que proporcionan tanto el PRODEP como el PEDPD, en gran parte se enfocan a incentivar la docencia. Además, ambos programas han sido creados en México por la Secretaría de Educación Pública (SEP), y por tanto, se derivan de los lineamientos propuestos a nivel nacional para el mejoramiento de la calidad educativa, vinculando así varios de sus objetivos con la política nacional, institucional e incluso con los del PIFD. Siendo así, la participación de los profesores en estos programas de incentivos debería contribuir al desarrollo de los objetivos planteados a nivel nacional en torno a la concepción de una mejora en la calidad educativa mediante el uso de TIC en la enseñanza, lo cual hace pertinente identificar en qué medida se ha logrado lo propuesto en el escenario de la UNISON.

\section{Resultados}

A continuación se describen los resultados obtenidos, organizados en función de las dimensiones establecidas para el análisis.

\subsection{La infraestructura tecnológica en la UNISON}

De acuerdo con la UNESCO (2006), uno de los aspectos importantes a considerar para la implementación de las tecnologías en la enseñanza, consiste en el análisis de las condiciones en que se encuentra la infraestructura tecnológica que se requiere implementar. Por tanto, se consideró pertinente indagar sobre las condiciones del equipamiento que se dispone en la institución; los resultados se muestran en la Figura 2.

Las condiciones de infraestructura son percibidas en buen estado, aunque solamente $5 \%$ de profesores las percibió como "extremadamente buenas". Esto se podría considerar como un resultado parcialmente positivo ya que la opción mayormente señalada indicó que las condiciones son "buenas". En este sentido, es pertinente indagar en torno a tipo de equipamiento en la institución y la disponibilidad de las TIC; es decir, ¿de qué tipo de equipamiento se dispone para innovar en la enseñanza? Por tanto, se les solicitó a los profesores señalar la disponibilidad de equipamiento en la institución. De acuerdo con los datos, las computadoras, el equipo de proyección y 
las áreas de trabajo con equipo de cómputo y acceso a Internet, es equipamiento que siempre se encuentra disponible en la institución, lo cual es positivo, pues se puede considerar como equipamiento básico (Figura 3).

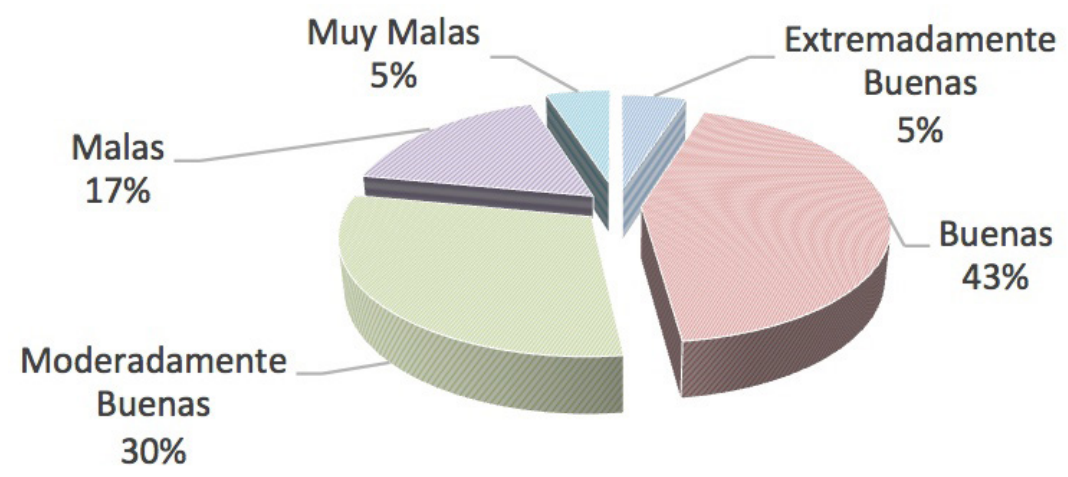

Figura 2. Condiciones de infraestructura tecnológica

Fuente: Elaboración propia.

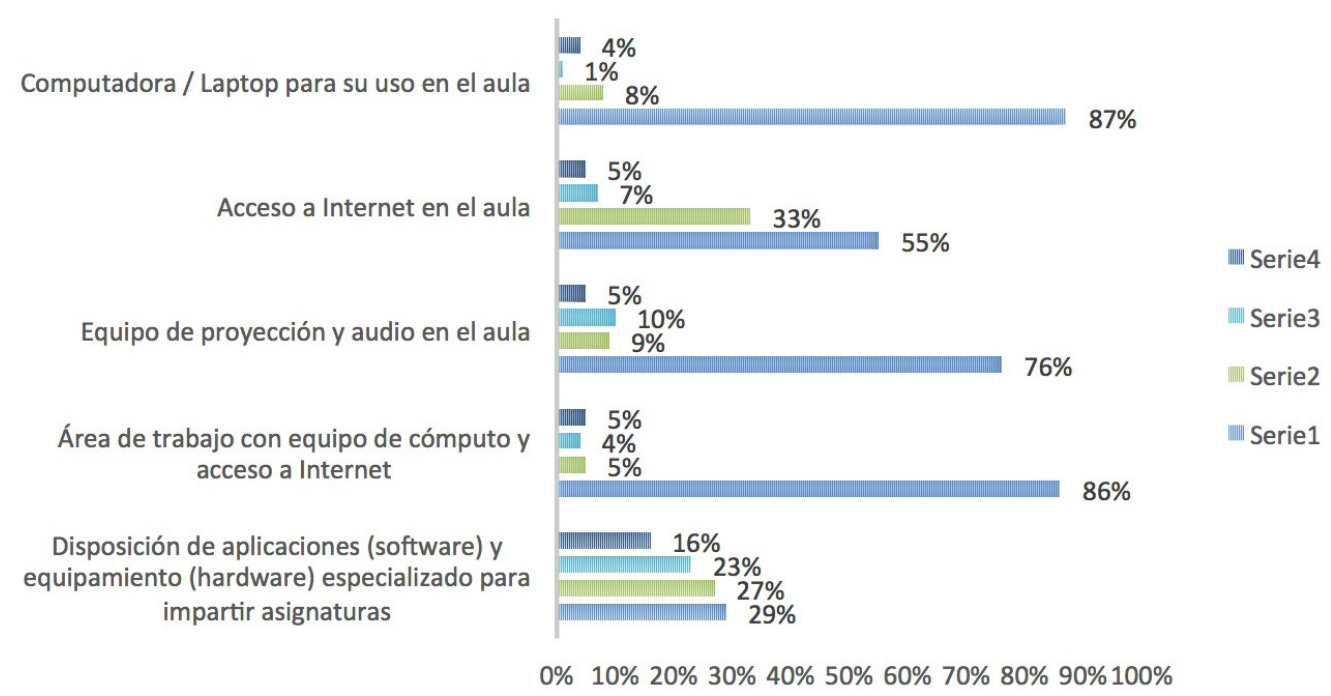

Figura 3. Equipamiento disponible en la institución

Fuente: Elaboración propia. 
Por otro lado, con respecto al equipamiento especializado, $56 \%$ de los profesores señaló tenerlo siempre y casi siempre disponible. Esto permite inferir que, a pesar del acceso que tienen los profesores a cierto equipamiento, las herramientas tecnológicas enfocadas a cuestiones especializadas de las asignaturas son insuficientes para casi la mitad de los profesores encuestados. De acuerdo con Casillas, Ramírez y Ortiz (2013) la forma de utilizar las tecnologías no es análoga, ya que existen brechas digitales en relación con la apropiación de las TIC según las disciplinas en que la infraestructura tecnológica se utilice. Por esta razón se consideró relevante analizar si la disponibilidad de equipamiento especializado varía entre cada disciplina, ya que al hablar de este tipo de equipamiento, las percepciones de los profesores con relación a la utilidad de las TIC para su ejercicio docente puede ser diversa; mientras que en unas disciplinas se puede considerar como muy necesaria la inclusión de alguna herramienta tecnológica especializada, en otras pueden no ser percibidas como algo tan pertinente y ser suficiente con el equipamiento básico (computadora, proyector e Internet).

Según los resultados, en las disciplinas adscritas al campo de las ciencias blandas (sociales, humanidades) el equipamiento especializado es percibido con mayor disponibilidad que en las disciplinas pertenecientes al campo de las ciencias duras (ingenierías, biológicas), ya que $43 \%$ de los profesores encuestados pertenecientes a ciencias duras consideró siempre tener disponible este tipo de equipamiento; por otro lado, 54\% de los profesores adscritos a disciplinas de ciencias blandas afirma tener siempre disponible el equipamiento especializado que necesita. Tal situación permite inferir una disponibilidad desigual de equipamiento especializado según el área de adscripción de los profesores en la UNISON.

En este contexto, es necesario observar el escenario de acuerdo con el planteamiento de Marcelo (2013), quien asegura que para generar una innovación en la enseñanza con el uso de TIC, el docente debe vincular el conocimiento sobre la asignatura con las tecnologías y la pedagogía. Esto hace evidente la necesidad de indagar sobre las posibilidades que consideran tener los docentes para vincular estos tres tipos de conocimiento. Es decir, ¿en qué medida la formación docente que reciben por parte de la institución permite a los docentes ampliar y vincular sus conocimientos sobre TIC, el contenido de las asignaturas y la pedagogía?

Debido a los intereses en mejorar la calidad de la enseñanza en la institución, habría que identificar si la infraestructura de la que se dispone se percibe como la requerida para impartir las asignaturas; también, si la formación institucional permite vincular los conocimientos de una manera productiva para el desarrollo de una mejor 
enseñanza; de lo contrario, la falta de equipamiento especializado se podría interpretar como un obstáculo y limitar el uso de Tic en el aula. En ese sentido, se les solicitó a los profesores señalar con qué frecuencia se presentan algunos obstáculos para el empleo de TIC en relación con la formación recibida. Se encontró que $64 \%$ de docentes considera algunas situaciones en que las oportunidades de formación ofrecidas por la institución son inadecuadas, además de que $53 \%$ también señaló que algunas veces falta claridad al plantear los beneficios de las TIC en estas capacitaciones (Figura 4). Esto permite cuestionar si verdaderamente el PIFD busca el desarrollo de habilidades tecnológicas en los profesores y beneficiar aspectos pedagógicos para la enseñanza de las asignaturas, posibilitando así el mejoramiento en el ejercicio de la docencia.

$$
\begin{gathered}
\text { Falta de claridad sobre los beneficios } \\
\text { de TIC } \\
\text { Inadecuadas oportunidades de } \\
\text { formación }
\end{gathered}
$$

Falta de tiempo para practicar cómo utilizar las TIC

Falta de tiempo en el horario de clase para utilizarlas en el aula

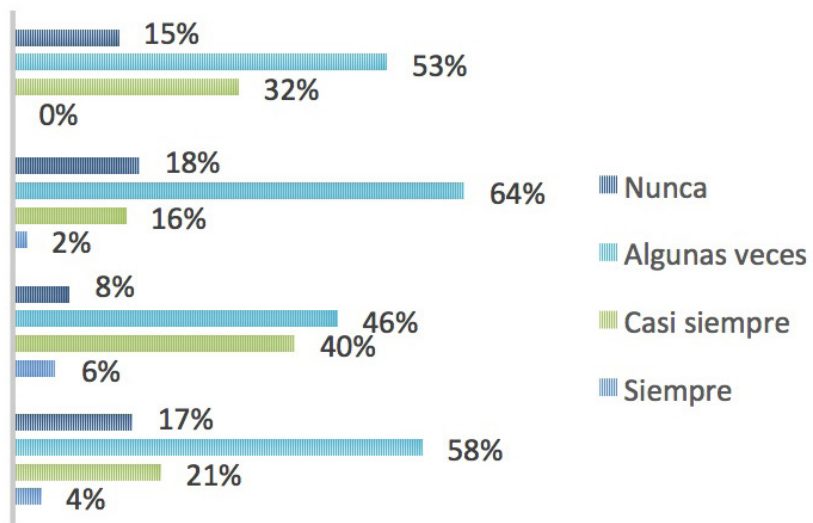

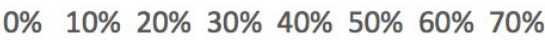

Figura 4. Obstáculos para el uso de TIC

Fuente: Elaboración propia.

Estos resultados permiten observar que las oportunidades de formación que ofrece la institución tienen una tendencia a considerarse algunas veces como inadecuadas por los profesores, por lo cual es pertinente indagar más sobre este punto.

Con respecto a la participación de hombres y mujeres en el PIFD, particularmente sobre la formación que es recibida en la institución para el uso de las TIC, se identificó que el apoyo pedagógico para el empleo de las TIC en la enseñanza es percibido de manera diferente entre hombres y mujeres. En relación con el apoyo pedagógico en- 
tre compañeros de la institución, el $75 \%$ de las mujeres consideró que siempre tienen apoyo suficiente, a diferencia de los hombres, quienes seleccionaron esta opción con una menor frecuencia ( $25 \%$ ). Esto indica que, para algunas mujeres, recibir apoyo para el empleo pedagógico de las Tic puede ser más accesible que para algunos profesores.

\subsection{Formación institucional docente}

Con relación a la formación docente para el uso de TIC, Swig (2015) afirma que las habilidades adquiridas por los profesores se pueden complementar con un proceso de apropiación personal y, por lo tanto, es importante que los docentes utilicen las TIC fuera del aula, lo que les permitiría la posibilidad de generar diferentes formas de percibir su capacitación.

Por otro lado, se le solicitó al docente señalar el nivel de capacitación que considera tener para el uso de TIC en la enseñanza, en el cual la mayoría señaló estar capacitado; sin embargo, 25\% indicó estar poco capacitado (Figura 5).

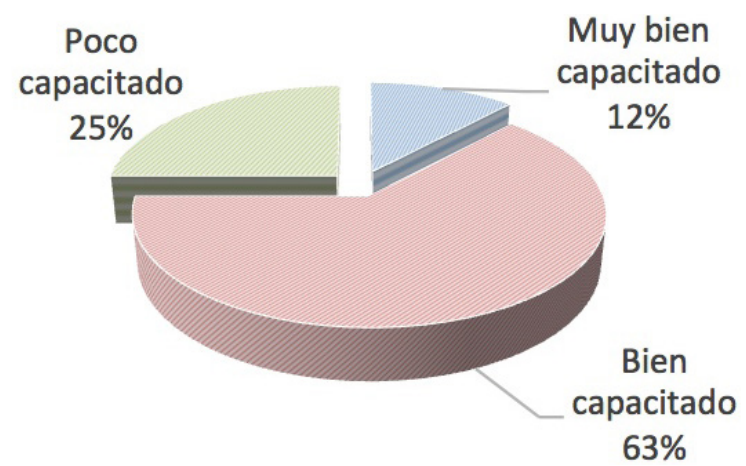

Figura 5. Nivel de capacitación del profesorado

Fuente: Elaboración propia.

Los resultados permiten inferir que la capacitación que reciben los docentes es considerada positiva; no obstante, esta opinión puede deberse a la formación recibida de diversas instituciones o por experiencia propia como docentes, y no necesariamente a la formación que la UNISON ofrece. En este sentido, se indagó sobre qué es lo que más ha incidido para los profesores en su formación sobre el uso de TiC (Figura 6). 


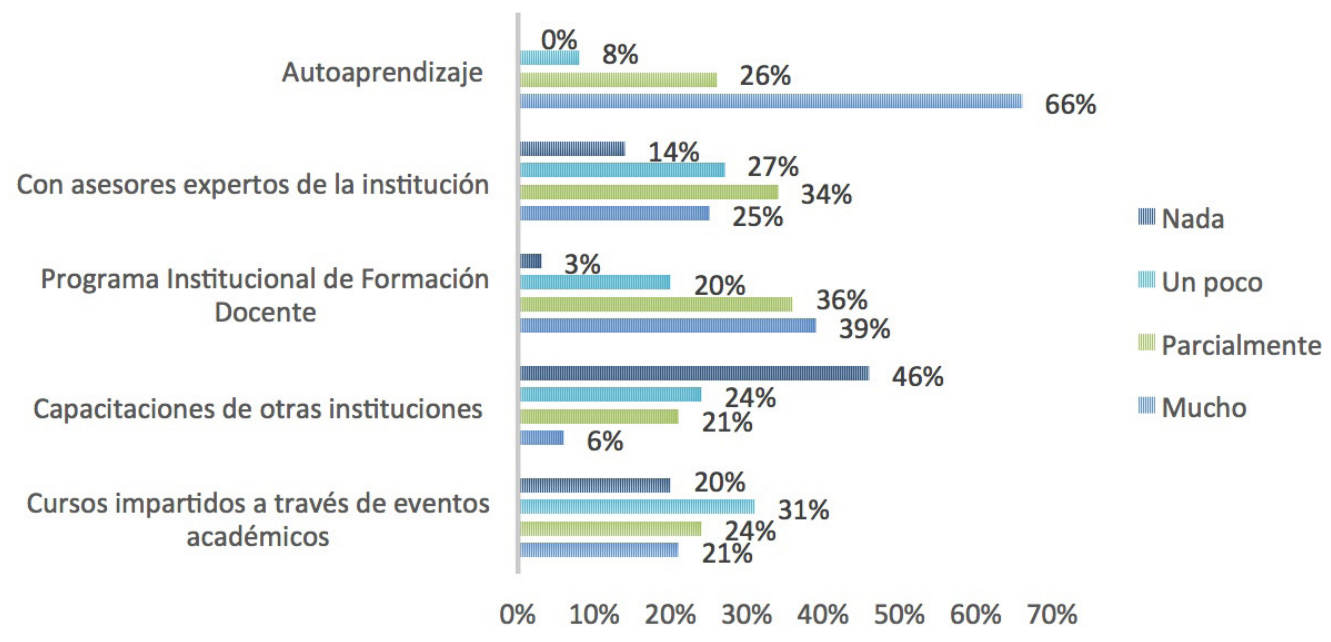

\section{Figura 6. Incidencias en la formación docente para el uso de TIC}

Fuente: Elaboración propia.

Aunque también se puede identificar que 39\% de los profesores consideró que el PIFD ha influido mucho en su formación para emplear las TIC en la enseñanza, un resultado más sobresaliente es que poco más de la mitad de los profesores señaló que la estrategia de autoaprendizaje es lo que ha incidido mucho en su formación sobre el uso de TIC.

Considerando el planteamiento de Rogers (2003) en torno a la relación de los participantes de un mismo sistema donde se promueva el desarrollo de innovaciones, se indagó si existen diferencias entre hombres y mujeres en cuanto a las opiniones de apoyo por compañeros al momento de requerir asistencia para el uso pedagógico de las TIC.

Tomando en cuenta que es la mayoría de los profesores quien considera que algunas veces son inadecuadas las oportunidades de formación en la institución, se retoman los resultados sobre los motivos de participación en el PIFD, es decir, ¿qué estimula a los profesores para participar en un programa de capacitación?

Como se puede observar en la Figura 7, más de la mitad de los docentes se sintió motivada para capacitarse, aparentemente, debido a las creencias de que al participar pueden lograr un aporte sustancial para mejorar la enseñanza en el aula. 


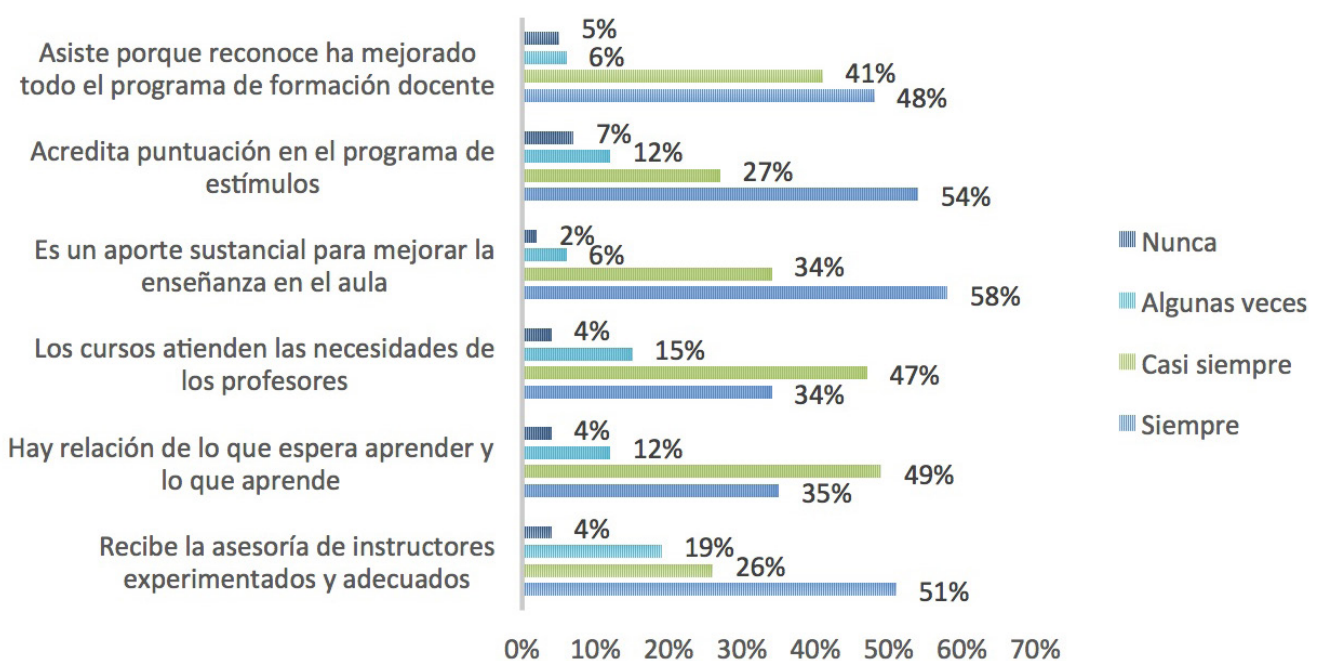

Figura 7. Motivos de participación en el PIFD

Fuente: Elaboración propia.

Esto coincide con lo expuesto por Prestridge (2012), quien afirma que las creencias personales son uno de los factores que influyen directamente en el interés de los docentes para habilitarse, además de concordar con la finalidad del programa, que busca formar profesores entusiastas y participantes en la capacitación mediante una reflexión sobre la utilidad de las TIC en el aula. Por tanto, se llevó a cabo un cruce de los resultados obtenidos para determinar diferencias entre los profesores que participan en programas de incentivos y los que no, con relación a sus motivos para capacitarse en el PIFD, con el propósito de lograr un acercamiento entre quienes verdaderamente pretenden mejorar su ejercicio docente mediante su capacitación para el uso de las TIC y su participación en programas de incentivos.

Los profesores que participan en programas que incentivan el desarrollo profesional (45), son quienes señalaron que su participación en el PIFD no se debe a una motivación para mejorar su enseñanza. Esto podría ser uno de los efectos adversos de los programas de incentivos, pues de acuerdo con Tearle (2003), para que los profesores puedan transformar su enseñanza a partir de la formación sobre el empleo de las TIC, se debe mostrar una actitud favorable que permita el aprendizaje y enfocar la atención en la obtención de nuevas habilidades a través de los cursos o la capacitación. 
Otro de los resultados que predominan, indica que las razones para participar en el programa de formación se debe a la puntuación que representa su capacitación para el programa de estímulos ( $54 \%$ señaló que siempre), lo cual despierta el interés en identificar las verdaderas razones del entusiasmo que genera el programa. Es decir, los resultados permiten identificar que se genera el interés en participar en la formación debido al estímulo que representan los puntos de las capacitaciones en los programas de incentivos al ejercicio docente. Asimismo, menos de la mitad (34\%) señaló que los cursos siempre atienden sus necesidades de formación; esto, aunado a las opiniones que en general se tienen de la incidencia del PIFD, pone en duda la pertinencia que el programa ha tenido para los profesores.

Por otra parte, se indagó sobre las creencias que se tienen sobre el empleo de las TIC en la enseñanza (Figura 8), ya que esto permite identificar la actitud de los profesores al utilizar las Tic en su ejercicio docente y capacitarse para esto. De acuerdo con los resultados, se puede inferir que, en la mayoría de los casos, se tiene una opinión favorable en cuanto a la utilidad de las tecnologías, lo cual implica que los profesores se encuentran dispuestos a implementar estrategias en el aula que mejoren los procesos de aprendizaje al incluir las TIC.

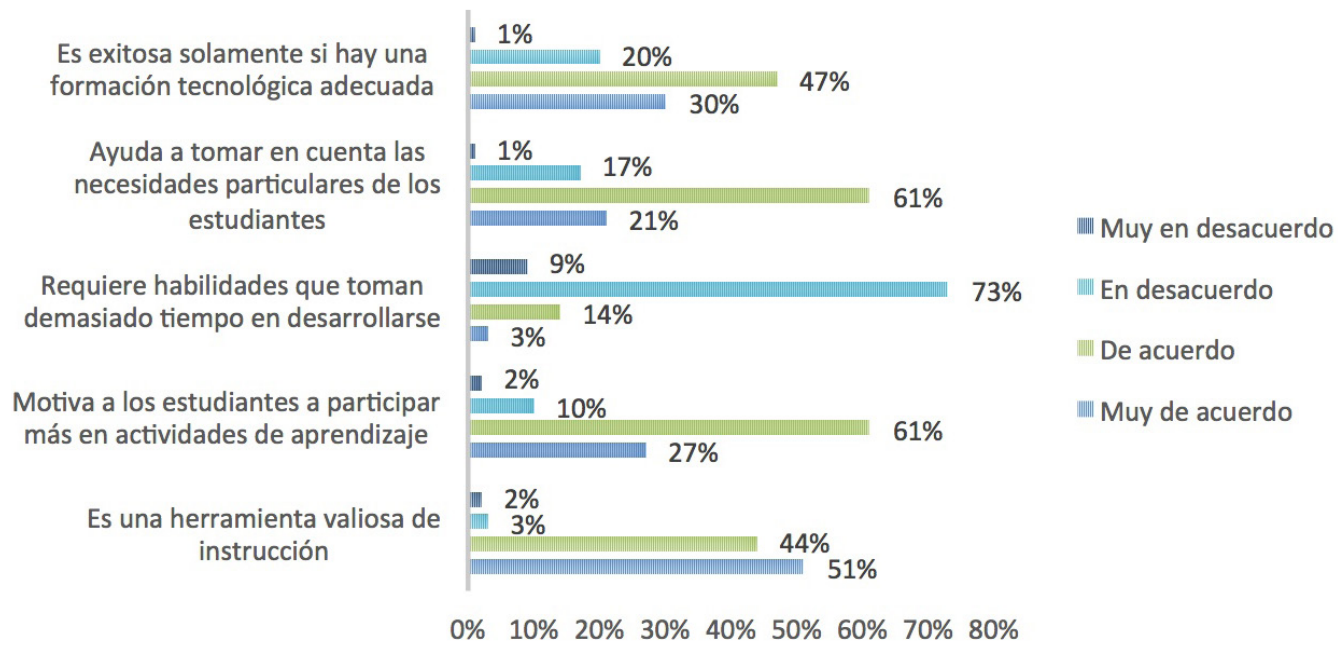

\section{Figura 8. Creencias sobre el empleo de TIC en la enseñanza}

Fuente: Elaboración propia. 
El empleo de las TIC en el aula no sólo implica utilizar las herramientas tecnológicas que están disponibles en la institución para instruir las clases de alguna asignatura, sino que requiere de una formación que vincule las habilidades tecnológicas para utilizar el equipamiento con las nociones necesarias para transmitir el conocimiento. Por lo tanto, el fomento del uso de TIC en la institución debe permitir mejoras en las habilidades tecnológicas que son requeridas por el profesorado para mejorar su ejercicio docente. Para esto, los profesores indicaron algunas actividades de apoyo a su ejercicio docente que desempeñan mediante el uso de las TiC (Figura 9).

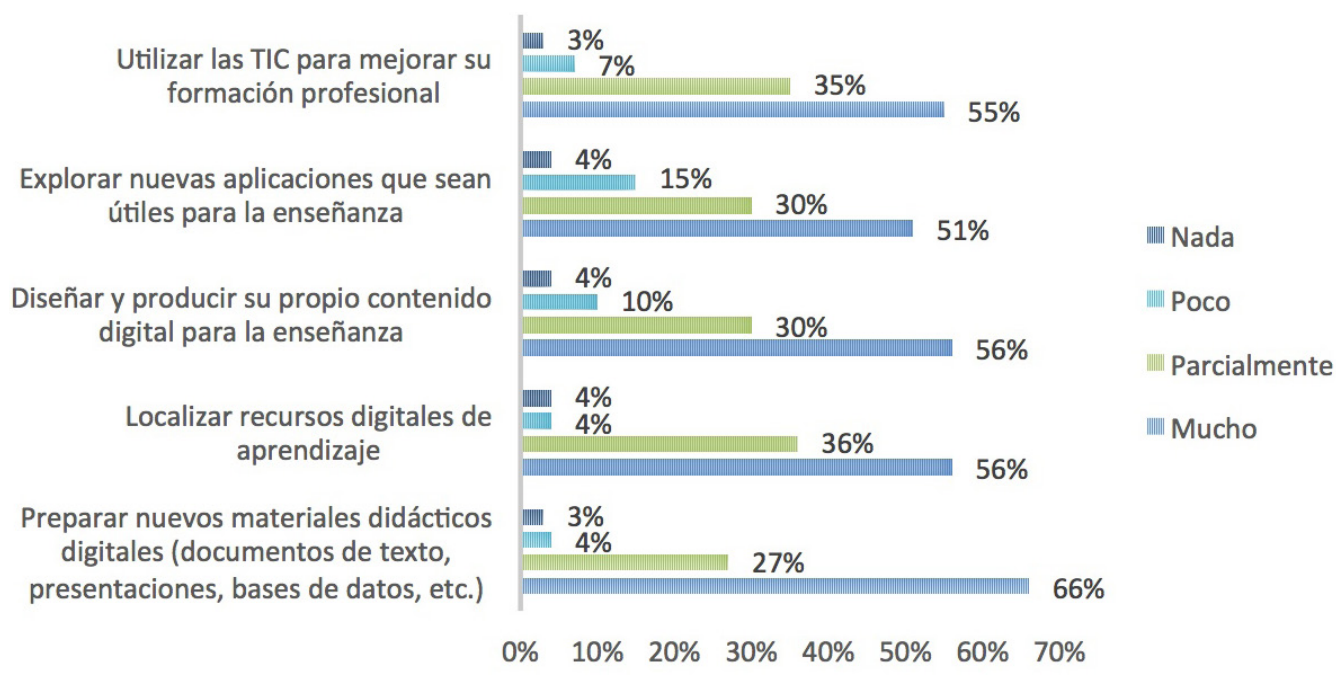

Figura 9. Actividades para la docencia mediante el uso de TIC

Fuente: Elaboración propia.

Los resultados indican que las tecnologías han apoyado a los profesores principalmente en la preparación de materiales didácticos en formato digital, los cuales facilitan o pueden transformar la práctica docente. Asimismo, más de la mitad de los profesores señaló que utiliza las tecnologías para localizar recursos digitales, explorar nuevas aplicaciones y diseñar contenido, además de admitir que mejoran su formación profesional al utilizarlas. 
Por otro lado, los profesores indicaron en qué tipo de cursos les interesa participar (Figura 10), como parte de un acercamiento para reconocer cuál es el interés general que se tiene para capacitarse y detectar necesidades de formación en el profesorado.

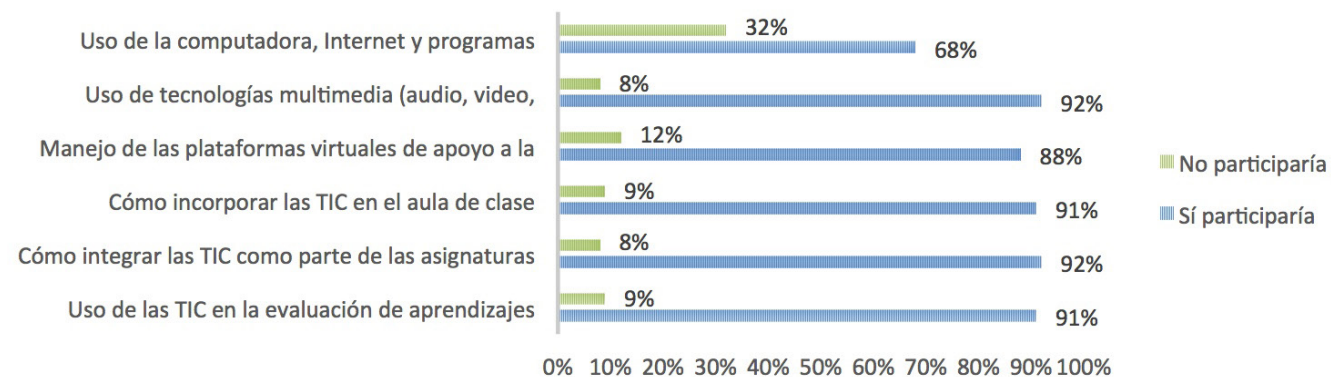

Figura 10. Interés por participar en capacitaciones sobre TIC según su tema

Fuente: Elaboración propia.

El 68\% de los profesores señala encontrarse más interesado en participar en cursos sobre el uso de la computadora e Internet y programas básicos. No obstante, más de $80 \%$ de los profesores advirtió encontrarse interesados en participar en cursos especializados y enfocados en la docencia. Estos resultados permiten inferir la pertinencia que debe representar para la institución el formar un vínculo entre los requerimientos de los docentes y los objetivos de sus políticas, al momento de planificar cursos de formación para mejorar la enseñanza mediante el empleo de TIC.

Otros aspectos a considerar consisten en poner a disposición de los profesores infraestructura tecnológica especializada con las asignaturas que imparten y proporcionar formación para el empleo en cuestiones pedagógicas. En ese sentido, se realizó una comparación de los profesores según la disciplina de su departamento de adscripción, para identificar quiénes prefieren los cursos sobre los temas que son básicos, como los relacionados con el uso de computadora, Internet y programas básicos (Figura 11). 


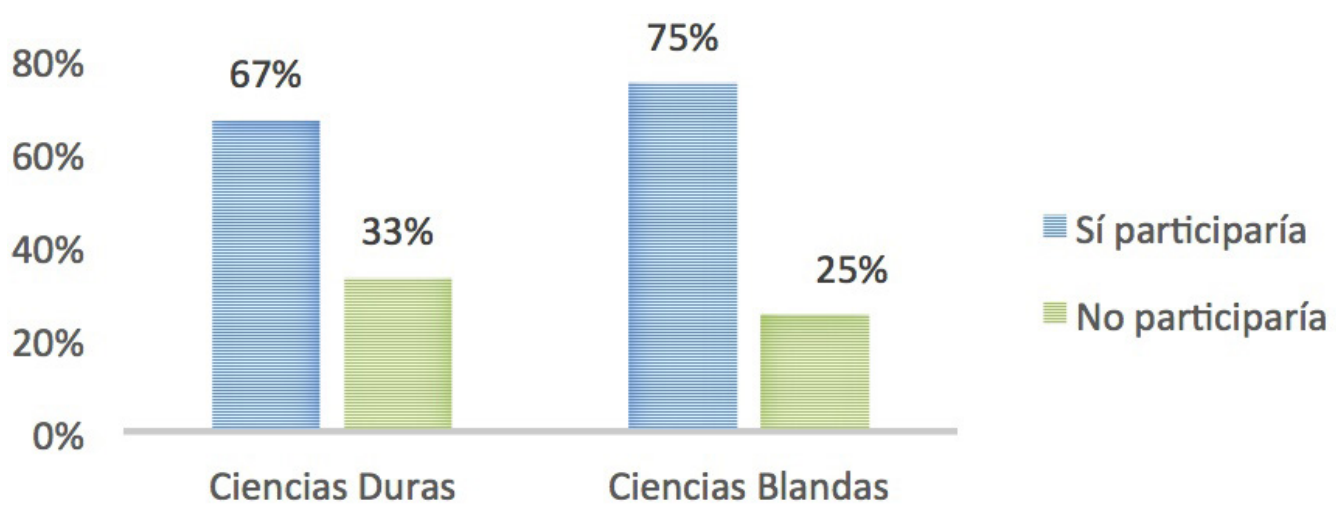

Figura 11. Interés por participar en cursos básicos sobre TIC según el tipo de disciplina

Fuente: Elaboración propia.

Se encontró que hay un menor interés de los profesores de ciencias duras para participar en cursos sobre algunas temáticas básicas, y un mayor interés de los profesores adscritos a departamentos de ciencias blandas para participar en este tipo de capacitaciones. Esto podría explicarse por el equipamiento que cada asignatura requiere; es decir, generalmente para los profesores de ciencias blandas el equipamiento tecnológico básico suele ser suficiente, mientras que para un profesor de ingeniería, la infraestructura tecnológica requerida podría ser más especializada.

A partir de los resultados arrojados, se puede inferir que la UnISON parece buscar una transformación mediante la implementación de políticas orientadas a integrar las TIC en el desarrollo del ejercicio docente de forma significativa, y por lo tanto, impactar en la percepción de los profesores en torno a cómo, para qué y cuándo usar el equipamiento tecnológico disponible.

Sin embargo, y a pesar de que algunos profesores han manifestado una mentalidad favorable para emplear las tecnologías en sus actividades académicas, en ocasiones la infraestructura tecnológica con la que cuentan no es necesariamente la requerida para sus asignaturas. Por lo que la participación en programas derivados de la política pública, como el PRODEP o el SNI, puede llegar a ser una salida para aquellos profesores que requieren equipamiento especializado y no lo tienen disponible por parte de la institución. Siendo así, se puede interpretar que las TIC se encuentran par- 
cialmente integradas en la UNISON, de tal manera que los profesores acuden al programa de formación docente que ofrece la institución con la intención de mejorar sus habilidades docentes, incluyendo el empleo de TIC; sin embargo, la percepción de la mayoría de los docentes que ha participado en el PIFD concibe a las oportunidades de formación de la institución como inadecuadas y en una escasa relación con sus necesidades de capacitación para emplear las TIC en cada asignatura.

En ese sentido, Careaga y Avedaño (2007) afirman que es necesario generar propuestas formativas enfocadas en el aspecto pedagógico y que constituyan un referente para garantizar el uso eficiente y significativo de las TIC en la educación universitaria, especificando qué habilidades tecnológicas y desempeños se esperan en el perfil de los profesores; así mismo, estas habilidades se deben encontrar enfocadas en atender las deficiencias que han sido detectadas por parte de los mismos profesores. Tal situación hace notar la pertinencia de tomar en cuenta las necesidades de los profesores al momento de plantear programas de formación docente, ya que para alcanzar mayores probabilidades de éxito en una institución educativa al momento de implementar un programa de formación docente sobre el uso de las TIC, al docente se le debe dotar de conocimientos relacionados entre sí, que vinculen los conocimientos requeridos para impartir su asignatura, los conocimientos referentes a la pedagogía y los conocimientos relacionados con el uso de la tecnología que se pretende implementar, y a su vez, estos conocimientos deben contemplar el contexto en el que la institución se desenvuelve (Marcelo, 2013).

\section{Conclusiones}

La tendencia del PIFD en la UNISON ha sido impulsar mejoras en el desarrollo de la enseñanza por medio de la concepción de una educación con calidad, que es planteada a partir de las sugerencias de la política nacional, donde se promueve la inversión en infraestructura tecnológica y la formación para el empleo de ésta en el aula; esto se ha buscado alcanzar en el escenario de la UNISON por medio del PDI 2013-2017 (UNISON, 2013). De tal forma que los profesores han llegado a acudir a este programa de formación con la intención de mejorar sus habilidades docentes incluyendo el empleo de TIC, pero en ocasiones las habilidades que buscan ser desarrolladas no llegan a ser las esperadas ni las pertinentes. Esto hace necesario generar propuestas formativas enfocadas en el aspecto pedagógico que constituyan un referente para garantizar 
el uso eficiente y significativo de las TIC en la educación universitaria, especificando qué habilidades tecnológicas y desempeños se esperan de los profesores para ser congruentes con las necesidades de formación docente.

Por lo tanto, se puede hablar del PIFD en la UNISON como una política implementada en la institución; sin embargo, los resultados obtenidos no permiten afirmar que la implementación haya sido exitosa o que haya alcanzado los objetivos que ha planteado, ya que según Berman (1993), una política bien implementada se alcanza cuando ésta logra una adopción mutua entre los individuos involucrados y lo propuesto por la política. En este caso, los docentes se encuentran interesados en recibir capacitación para el uso de TIC en la enseñanza, sin embargo, el aprendizaje que se les ofrece por parte del PIFD no ha logrado atender todas las necesidades de los profesores como para que ellos consideren que la formación que reciben por la institución es lo que más influye para que utilicen las tecnologías en virtud de una mejor enseñanza.

Por otro lado, y en relación con lo expuesto por Fullan (2002) sobre la pertinencia del cambio significativo en los profesores, se interpreta el escenario de éstos como en la etapa de aceptación, ya que se encuentran comprometidos con los cambios y están involucrados en participar en el programa de formación que se ha implementado. Sin embargo, no parece ser claro el rumbo que tiene el cambio que se espera concebir en los profesores por medio del PIFD.

También es evidente que la formación institucional ha incidido en forma limitada, o al menos no lo suficiente, para una parte importante de profesores, $y$ en algunos casos se ha llegado a ocasionar que el interés en utilizar las tecnologías se pierda. Esto permite suponer un escenario institucional complejo, con objetivos no logrados en su totalidad en torno a las motivaciones que se busca generar en los docentes para desarrollar competencias tecnológicas y crear las condiciones para que se empleen las tecnologías en la enseñanza; también es un aspecto que permea el cumplimiento de metas institucionales que se asemejan a las establecidas en la política nacional para mejorar la calidad de la enseñanza.

A pesar de todas las situaciones identificadas, la unison debe enfrentar los retos y generar un escenario favorecedor para la enseñanza innovadora por medio del desarrollo de las habilidades tecnológicas a través el PIFD, ya que a pesar de que asegura buscar una educación de calidad, no se ha propuesto capacitar ni a la mitad de sus profesores; incluso, si se solucionaran los problemas en la formación que se ofrece, aún se necesitaría generar metas que aspiren a capacitar a una mayor cantidad de profesores. 
Si la formación institucional para el uso de TIC se otorga a una mayor cantidad de profesores adscritos a la Universidad, se podrá aumentar la posibilidad de cumplir con los objetivos planteados por la institución al momento de invertir en infraestructura tecnológica, siempre y cuando la formación atienda las necesidades de los profesores para emplear las TIC en sus asignaturas. De no atenderse así, el mejoramiento de la enseñanza mediante la formación seguirá permaneciendo como un objetivo lejano.

Finalmente, este análisis brinda una visión del escenario institucional que permite un acercamiento desde las opiniones de los docentes; no obstante, es pertinente complementar este estudio a través de análisis cualitativos que permitan profundizar en la opinión de los profesores en torno a la inversión que las IEs hacen en infraestructura tecnológica y sobre lo que se logra en la enseñanza mediante la formación institucional docente, ya que esto permitirá establecer deficiencias y, a su vez, proporcionar recomendaciones que favorezcan la innovación educativa mediante TIC en la enseñanza de las universidades.

\section{Lista de referencias}

Altbach, P. G. (2008). Funciones complejas de las universidades en la era de la globalización. En Global University Network for Innovation, Report higher education in the world (pp. 5-19). Madrid: Mundi Prensa.

Berman, P. (1993). El estudio de la macro y la micro implementación. En L. F. Aguilar (Coord.), La implementación de las políticas públicas (pp. 125-159). México: Porrúa.

Borbón, I. (2015). Formación y práctica del docente en la implementación de la educación virtual de la Universidad de Sonora (Tesis inédita de maestría). Universidad de Sonora, México.

Careaga, M., \& Avendaño, A. (2007). Estándares y competencias TiC para la formación inicial de profesores. REXE. Revista de Estudios y Experiencias en Educación, 6(12), 93-106. Recuperado de www.rexe.cl/ojournal/index.php/rexe/article/view/185

Casillas, M. A., Ramírez, A., \& Ortiz, V. (2013). El Capital Tecnológico una nueva especie del capital cultural: Una propuesta para su medición. Trabajo presentado en el XII Congreso Nacional de Investigación Educativa del Consejo Mexicano de Investigación Educativa, Guanajuato, México. 
Corbetta, P. (2007). Metodología y técnicas de investigación social. Madrid: McGrawHill.

Correa, J. M., \& Martínez, A. (2010). ¿Qué hacen las escuelas innovadoras con la tecnología?: Las TIC al servicio de la escuela y la comunidad en el colegio Amara Berri. Teoría de la Educación. Educación y Cultura en la Sociedad de la Información, 11(1), 230-261. Recuperado de http://www.redalyc.org/articulo. oa? id=201014897010

Ducoing, P. (2003). Sujetos, actores y procesos de formación (Tomo II). México: Consejo Mexicano de Investigación Educativa.

Ducoing, P., \& Fortoul, B. (2013). Procesos de formación (Vol. II). México: Consejo Mexicano de Investigación Educativa.

European Union. (2013). Survey of Schools: ICT in Education. Benchmarking Access, use and attitudes to technology in Europe's Schools. Final Report. Brussels: European Schoolnet. Recuperado de https://ec.europa.eu/digital-single-market/sites/ digital-agenda/files/KK-31-13-401-EN-N.pdf

Fullan, M. (2002). El significado del cambio educativo: Un cuarto de siglo de aprendizaje. Profesorado. Revista de Currículum y Formación del Profesorado, 6(1-2). Recuperado de https://recyt.fecyt.es/index.php/profesorado/article/view/41935

Gobierno de la República. (2013). Plan Nacional de Desarrollo 2013-2018. Recuperado de http://pnd.gob.mx/wp-content/uploads/2013/o5/PND.pdf

Grijalva, H. (2012). Informe anual 2011-2012 (Informe No. 3). Hermosillo, México: Universidad de Sonora. Recuperado de http://www.uson.mx/paginadelrector/informes/informe2012-2013.pdf

Leyva, A. G. (2015). Capital Tecnológico en profesores de la Universidad de Sonora (Tesis de maestría inédita). Universidad de Sonora, México.

López, M. C. (2013). Impacto de las tecnologías de la información y la comunicación (тIC) en el docente universitario. El caso dela Universidad de Guadalajara. Perspectiva Educacional, Formación de Profesores, 52(2), 4-34. doi:10.4151/o7189729Vol.52-Iss.2-Art.180

López, R., \& Ramírez, A. (2016). Políticas de equipamiento tecnológico en Educación Superior: reflexiones y orientaciones. Debate Universitario, 5(9), 53-67. Recuperado de http://portalreviscien.uai.edu.ar/ojs/index.php/debate-universitario/article/view/v5ngao4

Marcelo, C. (2013). Las tecnologías para la innovación y la práctica docente. Revista Brasileira de Educação, 18(52), 25-47. Recuperado de http://www.scielo.br/ 
scielo.php?pid=S1413-24782013000100003\&script=sci_abstract\&tlng=es

Moral del, M. E., \& Villalustre, L. (2012). Didáctica universitaria en la era 2.0: competencias docentes en campus virtuales. Revista de Universidad y Sociedad del Conocimiento, 9(1), 36-50. Recuperado de http://www.redalyc.org/ pdf $/ 780 / 78023415004 . p d f$

Organización de las Naciones Unidas para la Educación, la Ciencia y la Cultura. (2004). Las tecnologías de la información y la comunicación en la formación docente. Guía de planificación. Uruguay: Autor, Recuperado de http://unesdoc. unesco.org/images/o012/001295/129533s.pdf

Organización de las Naciones Unidas para la Educación, la Ciencia y la Cultura. (2006). La integración de las Tecnologías de la Información y la Comunicación en los Sistemas Educativos. Buenos Aires: Instituto Internacional de Planeamiento de la Educación-Organización de las Naciones Unidas para la Educación, la Ciencia y la Cultura. Recuperado de http://unesdoc.unesco.org/ images/0015/001507/150785s.pdf

Organización para la Cooperación y el Desarrollo Económico. (2002). Los Desafíos de las Tecnologías de la Información y las Comunicaciones. España: Autor.

Prestridge, S. (2012). The beliefs behind the teacher that influences their Iст practices. Computers \& Education, $58(1)$, 449-458. Recuperado de http://www.sciencedirect.com/science/article/pii/So360131511002065

Ramírez, A., \& Casillas, M. A. (2014). Háblame de Tic. Tecnología Digital en Educación Superior. Córdoba, Argentina: Brujas.

Rogers, E. (2003). Diffusion of Innovations. Nueva York: The Free Press.

Salado, L., Velázquez, M., \& Ochoa, R. (2014). El capital tecnológico y el ejercicio docente: el caso de la Universidad Estatal de Sonora. Trabajo presentado en el Congreso Iberoamericano de Ciencia, Tecnología, Innovación y Educación de la Universidad de Buenos Aires, Argentina. Recuperado de https://www.oei.es/ historico/congreso2014/memoriactei/1140.pdf

Sautu, R., Boniolo, P., Dalle, P., \& Elbert, R. (2005). Manual de Metodología de Investigación. Construcción del marco teórico, formulación de los objetivos y elección de la metodología. Buenos Aires: Lumiere.

Swig, S. (2015). TICs y formación docente: formación inicial y desarrollo profesional docente. Washington, DC: Inter-American Dialogue. Recuperado de https://www. oas.org $/$ cotep $/$ GetAttach.aspx?lang=en \&cId=265\&aid=417

Tamayo, M. (2011). El proceso de la investigación científica. México: Limusa. 
José Ricardo López Espinosa, Edgar Oswaldo

González Bello, Rocío López González

Tearle, P. (2003). Enabling Teachers to Use Information and Communications Technology for Teaching and Learning through Professional Development: influential factors. Teacher Development, 7(3), 457-472. Recuperado de http:// www.tandfonline.com/doi/pdf/10.1080/13664530300200209

Torres, S., \& Barona, C. (2012). Los profesores universitarios y las TIC: uso, apropiación, experiencias. México: Juan Pablos/Universidad Autónoma del Estado de Morelos.

Universidad de Sonora. (2013). Plan de Desarrollo Institucional 2013-2017. Hermosillo, México. Recuperado de http://www.uson.mx/institucional/pdi2013-2017.pdf Universidad de Sonora. (2015). Programa Institucional de Desarrollo y Actualización de Habilidades Docentes. Hermosillo, México: Universidad de Sonora-Dirección de Innovación Educativa. 